\title{
Editorial
}

\section{Recent Advances on Modeling, Control, and Optimization for Complex Engineering Systems}

\author{
Kang Li, ${ }^{1}$ Guido Maione, ${ }^{2}$ Minrui Fei, ${ }^{3}$ and Xingsheng Gu${ }^{4}$ \\ ${ }^{1}$ School of Electronics, Electrical Engineering and Computer Science, Queen's University Belfast, Ashby Building, \\ Stranmillis Road, Belfast BT9 5AH, UK \\ ${ }^{2}$ Dipartimento di Ingegneria Elettrica e dell'Informazione (DEI), Politecnico di Bari, Via E. Orabona 4, 70125 Bari, Italy \\ ${ }^{3}$ School of Mechatronic Engineering and Automation, Shanghai University, 149 Yanchang Road, Shanghai 200072, China \\ ${ }^{4}$ School of Information Science and Engineering, East China University of Science and Technology, 130 Meilong Road, \\ Shanghai 200237, China \\ Correspondence should be addressed to Kang Li; k.li@qub.ac.uk
}

Received 21 December 2014; Accepted 21 December 2014

Copyright (C) 2015 Kang Li et al. This is an open access article distributed under the Creative Commons Attribution License, which permits unrestricted use, distribution, and reproduction in any medium, provided the original work is properly cited.

The last decade has seen a radical step-change in the scale and complexity of engineering systems, from industries like petrochemical, pharmaceutical, light industry, and machinery manufacturing, to power and energy system and transport, and so forth. Complexity arises from a number of factors, such as the nonlinear coupling among units and variables as well as the uncertainty introduced into the system. Further, the rapid progress of information and communication technologies makes the connections even more complicated and widespread. As the core technologies in dealing with complex systems, the development of new modelling, control, and optimization techniques for large-scale and complex engineering systems have attracted an increasing interest, and it becomes a multidiscipline theme bringing together the modern control theory, computer modelling, intelligent optimization, powerful real-time parallel computing, and networking technology.

The main focus of this special issue is on the new theories and their applications in modeling, control, and optimization for complex engineering systems, especially in industry applications. The topics of these papers cover advanced simulation, modelling, compensation, control, and optimization methods for complex systems and processes; networked control system theory and applications; planning, scheduling, and management; power electronics and power drives; power system operation and control with integration of renewables; electrical machinery and electrical apparatus; smart grid; intelligent transport systems and electric vehicles; wireless networks and sensors; fuzzy and neural systems and networks; metaheuristic algorithms and applications; data fusion and classification; advanced image processing technologies; and intelligent design.

These papers only serve as an introduction to the recent advances in modeling, simulation, control, and optimization for complex engineering systems and applications. It is hoped that this issue will serve as a catalyst for future research aimed at tackling complex engineering problems using advanced techniques.

\section{Acknowledgments}

We would like to express our deepest gratitude to the many reviewers who have helped with the review process for this special issue and the authors for their efforts in contributing the papers.

Kang $\mathrm{Li}$

Guido Maione

Minrui Fei

Xingsheng $G u$ 


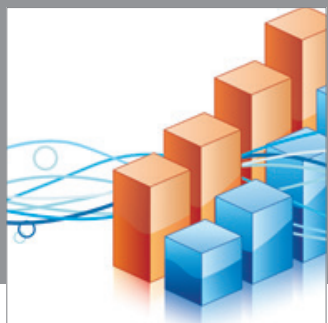

Advances in

Operations Research

mansans

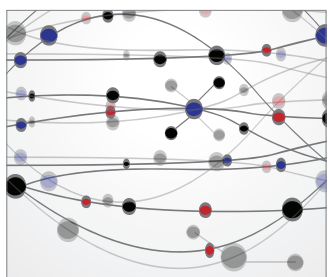

The Scientific World Journal
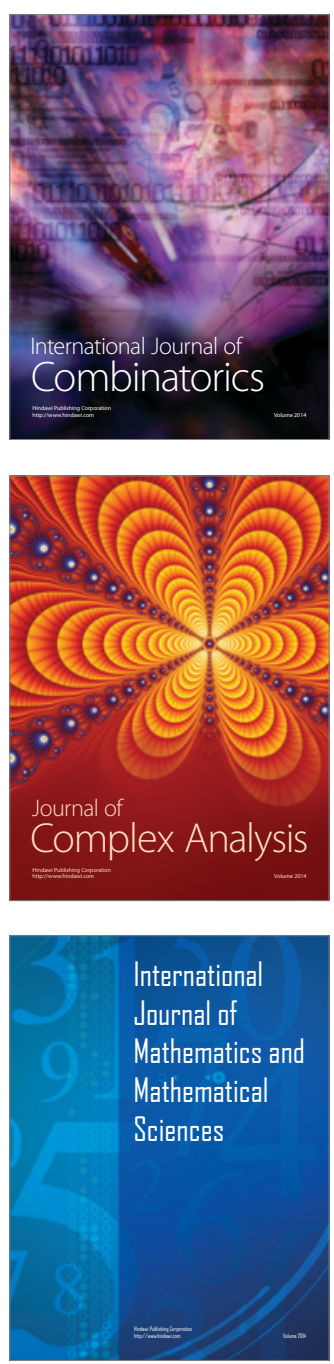
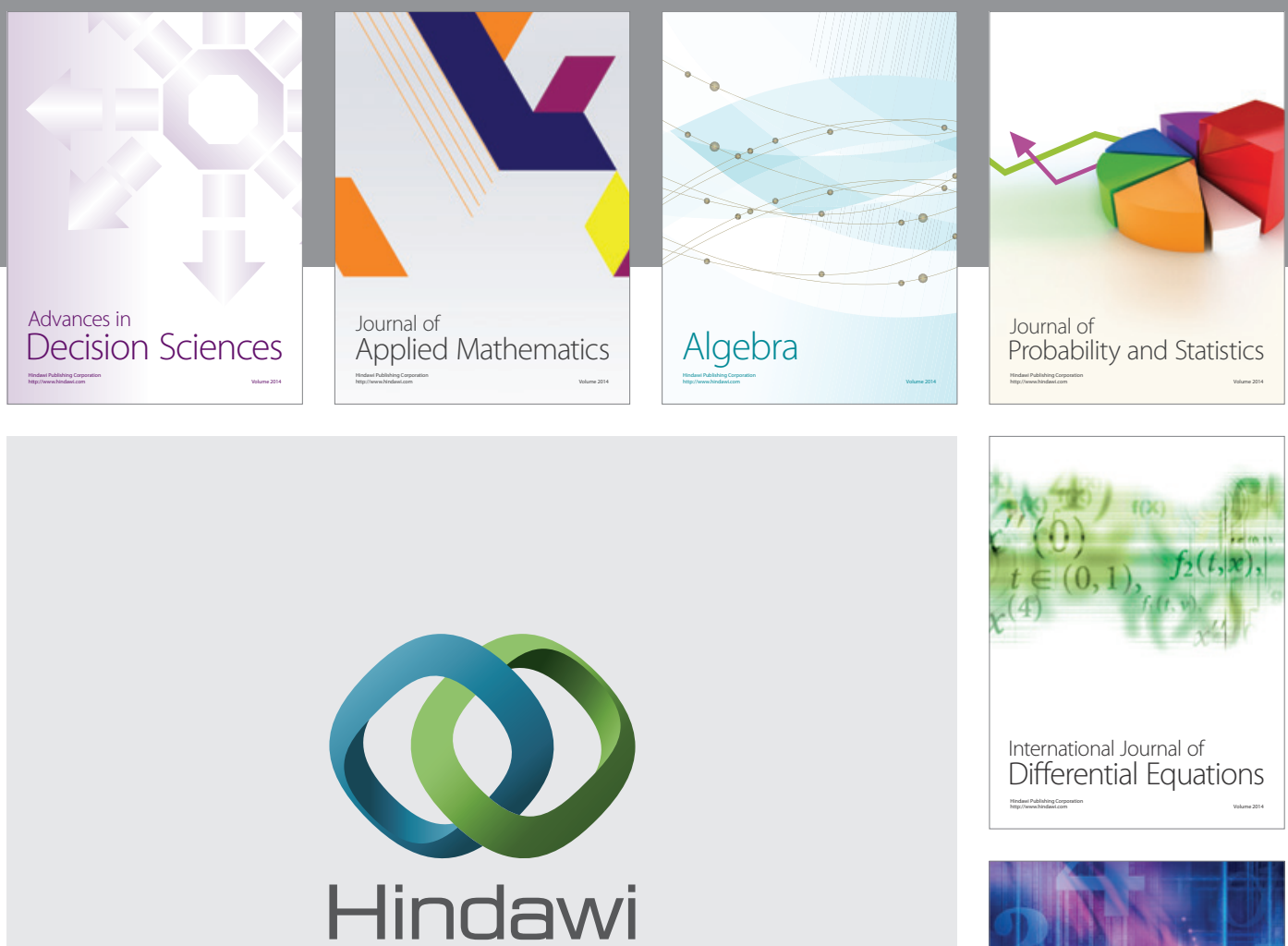

Submit your manuscripts at http://www.hindawi.com
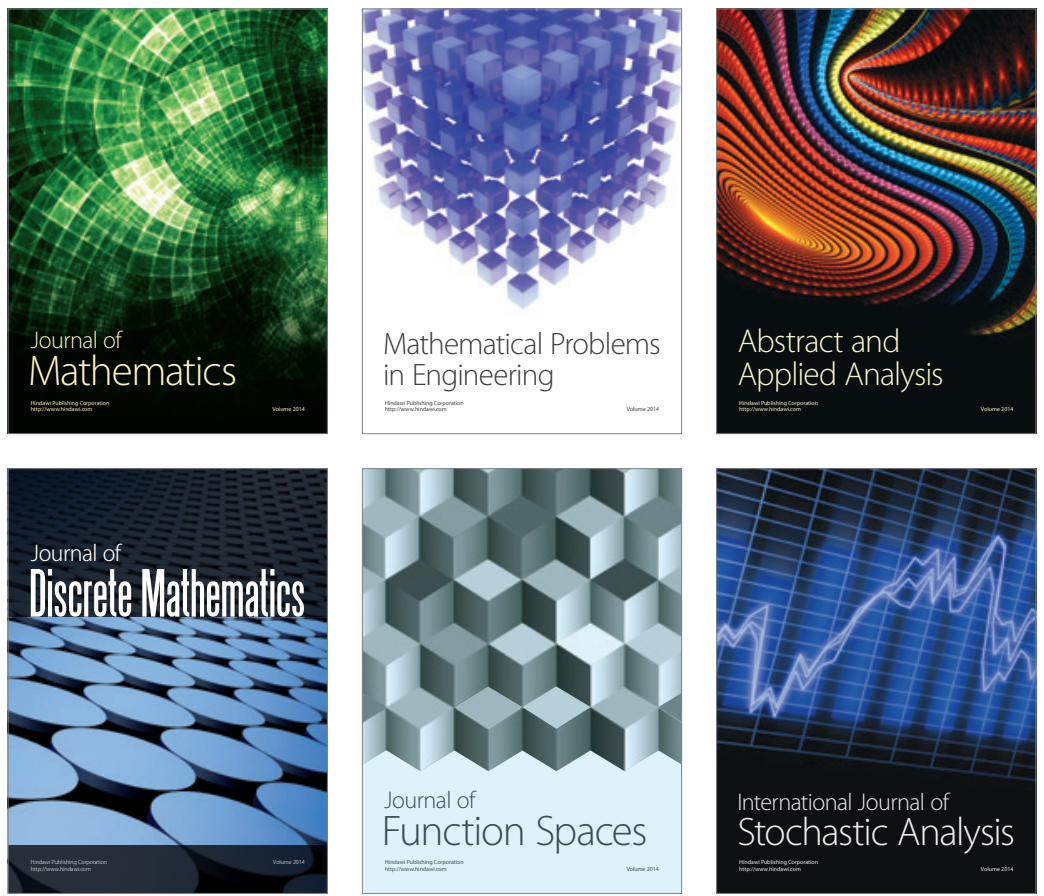

Journal of

Function Spaces

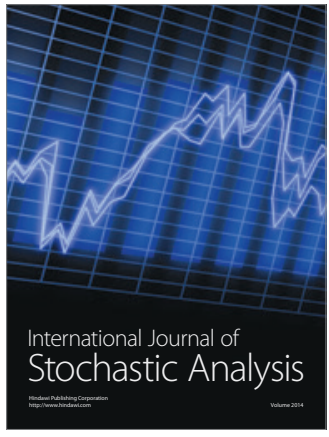

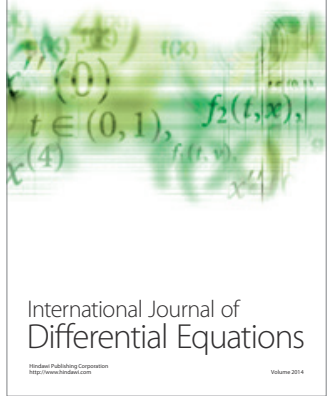
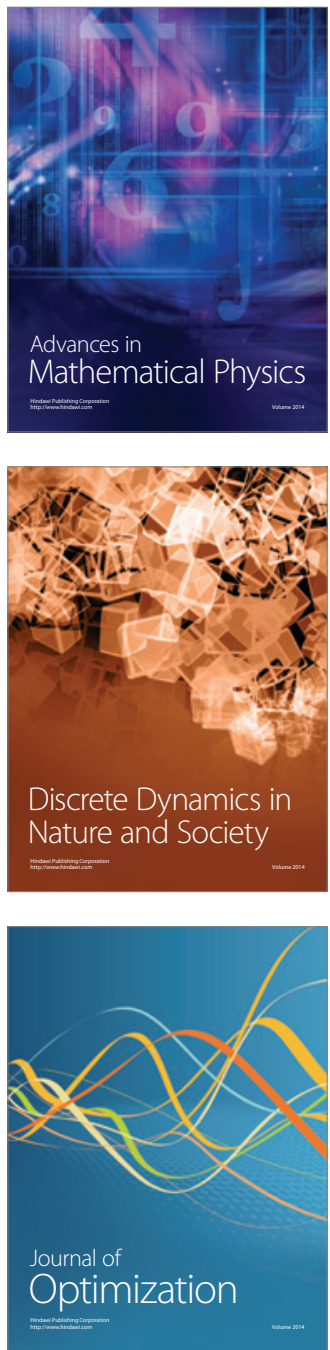